\title{
Dynamic deformation and fracturing properties of concrete under biaxial confinements
}

\author{
Huachuan Wang ${ }^{1}$, Kai Liu $^{2}$, and Qianbing Zhang ${ }^{1}$ \\ ${ }^{1}$ Monash University, Department of Civil Engineering, Clayton, VIC 3800, Australia \\ ${ }^{2}$ University of Oxford, Department of Engineering Science, Oxford, OX1 3PJ, United Kingdom
}

\begin{abstract}
The study of deformation and fracturing properties of concrete is essential to understand failure mechanisms of concrete material, especially under extreme and complex loading conditions, e.g. high strain rates and multiple confinements. In this study, dynamic deformation and fracturing behaviour of ordinary concrete under biaxial confinements are investigated by using a triaxial Hopkinson bar system, three-dimensional digital image correlation, and synchrotron X-ray computed tomography techniques. Results show that compressive strain localisation areas appear around aggregates due to the elastic difference between aggregate and matrix, where accompanied with initiation of interfacial cracks and propagation of main cracks. Transgranular cracks can also be observed in the central of specimen in $\mathrm{CT}$ slices due to the effect of strain concentration. In addition, CT slices with distinct properties in various directions indicate the anisotropic fracturing behaviour of concrete due to the effect of biaxial confinements.
\end{abstract}

\section{Introduction}

Concrete structures (e.g. tunnel linings, column-beam joints, and nuclear safety shells) under biaxial stress states are likely to suffer dynamic loads such as earthquake, impact, and blast in the service. Under these extreme loading conditions, concrete materials are more prone to deterioration and failure, with deformation and fractures significantly different from what has been observed under quasi-static conditions.

In order to study the dynamic behaviour of concrete under biaxial confinements, different experimental facilities have been applied, like hydraulic biaxial system [1], split Hopkinson bar with hydraulic jack [2], and triaxial Hopkinson bar (Tri-HB) system [3]. Compared with other facilities, the Tri-HB system could achieve the measurement of lateral stress wave histories during dynamic loadings [4, 5], which has been validated and replicated by numerical modelling [6]. Besides, to measure the deformation and crack opening displacement of specimens, strain gauges and resistance wire extensometers have been widely used in dynamic experiments [7]. In recent years, with the development and application of high-speed camera, the digital image correlation (DIC) technique is widely applied to dynamic tests to capture the full-field deformation and real-time fracturing process [8]. Compared with 2D-DIC, 3D-DIC technique can achieve the reconstruction of 3D shape and measurement of 3D deformation, which could describe the actual deformation process more precisely [9]. However, DIC technique can only obtain the surface deformation but 
hardly identify the internal fractures. Hence, micro-computed tomography ( $\mu$-CT) technique is promising to non-destructively reconstruct the structures and fractures within the sample.

In this study, dynamic tests with biaxial compressive pre-stress $(30,10) \mathrm{MPa}$ and impact velocity $16 \mathrm{~m} / \mathrm{s}$ are performed to study the deformation and fracturing properties of ordinary concrete. A simplified matrix-inclusion specimen is also used for verification. The Tri-HB system, 3D-DIC and synchrotron CT techniques were applied to achieve the measurement of mechanical and fracturing properties.

\section{Sample preparation}

Concrete sample fabricated in this study is composed of deionized water, ordinary Portland cement (OPC), river sand with the maximum size of $1.12 \mathrm{~mm}$ and basalt aggregate with the maximum size of $7 \mathrm{~mm}$ in proportions of $0.6: 1: 2: 3$ by weight. Basalt is adopted as coarse aggregate since its density is higher than other common aggregates and can be well distinguished from matrix in CT images with obvious density difference. The specimens are cast in steel moulds, and grinded into cubes with the size of $50 \mathrm{~mm}$ and flatness tolerance of $0.02 \mathrm{~mm}$ as shown in Figure 1. The composition and quasi-static compressive strength of the sample is listed in Table 1.

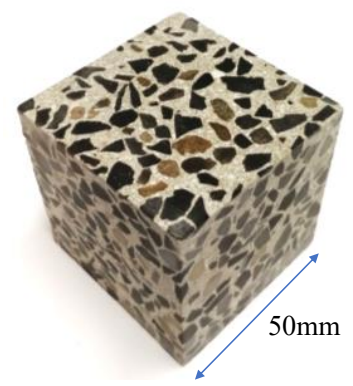

Fig. 1. Concrete sample.

Table 1. Composition and mechanical properties of concrete.

\begin{tabular}{|c|c|}
\hline Properties & Value \\
\hline Cement $\left(\mathrm{kg} / \mathrm{m}^{3}\right)$ & 356 \\
\hline Water $\left(\mathrm{kg} / \mathrm{m}^{3}\right)$ & 214 \\
\hline Sand $\left(\mathrm{kg} / \mathrm{m}^{3}\right)$ & 712 \\
\hline Aggregate $\left(\mathrm{kg} / \mathrm{m}^{3}\right)$ & 1068 \\
\hline Mean Density $\left(\mathrm{kg} / \mathrm{m}^{3}\right)$ & 2350 \\
\hline Compressive strength $(\mathrm{MPa})$ & 45.0 \\
\hline
\end{tabular}

\section{Experimental methods}

The dynamic compression tests of concretes under biaxial confinements were conducted on the the Tri-HB system (Figure 2.a). It consists of a servo-controlled hydraulic loading system to apply the quasi-static multiaxial confining pressures and a dynamic loading system to apply additional dynamic loading on the specimen. The experimental setup with a 3D-DIC technique for dynamic biaxial compression is shown in Figure 2.b. Two high-speed cameras (Phantom V2511) were installed at generally the same height for similar scope of views. Speckle pattern spraying, stereo calibration and camera synchronization verification were 
carried out firstly before dynamic tests. Details of the calibration and operation of Tri-HB system and 3D-DIC system are given in the literatures $[10,11,12]$.

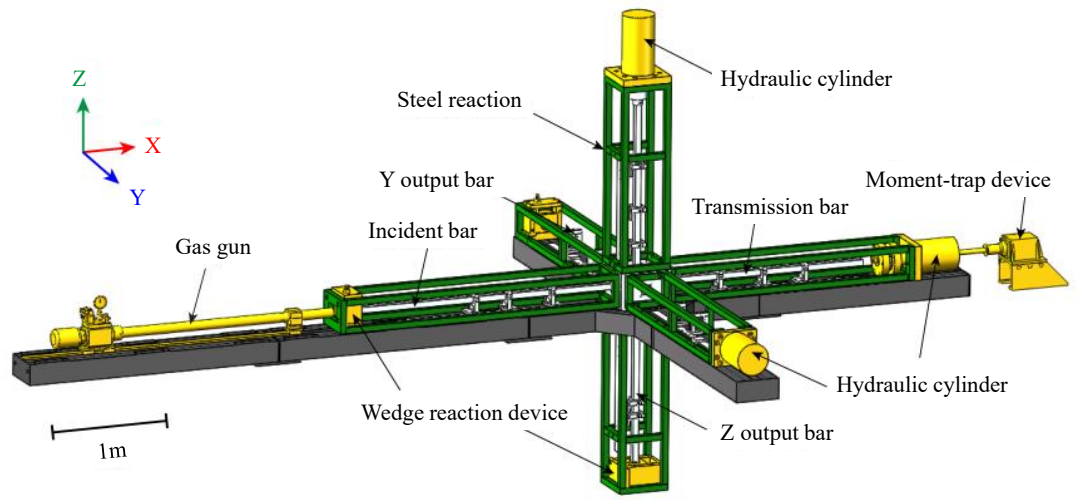

(a)

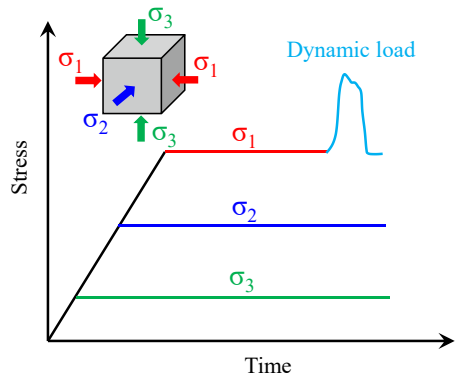

(b)

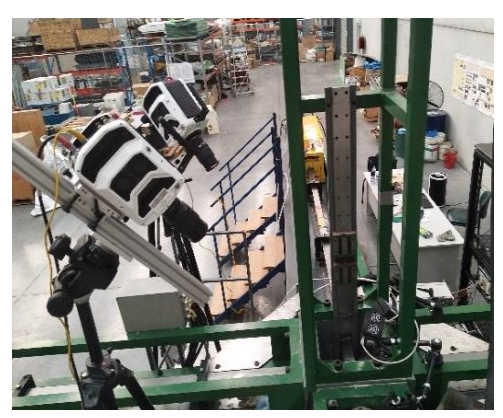

(c)

Fig. 2. (a) Triaxial Hopkinson bar system [10], (b) a typical loading path [11] and (c) 3D-DIC system by two high-speed cameras [12].

X-ray computed tomography (CT) system at Australian Synchrotron is adopted to capture internal fractures of post-failure specimens. As shown in Figure 3, X-ray beam is emitted from synchrotron light source and passes through the specimen (placed on rotation platform) to the detector. Compared with laboratory-based micro-CT, synchrotron light source extracts a monochromatic X-ray beam with higher photon flux, which significantly increases scanning speed and improves image quality $[13,14]$. The obtained CT images with resolution $17.8 \mu \mathrm{m}$ are further reconstructed with software Avizo 9.5.

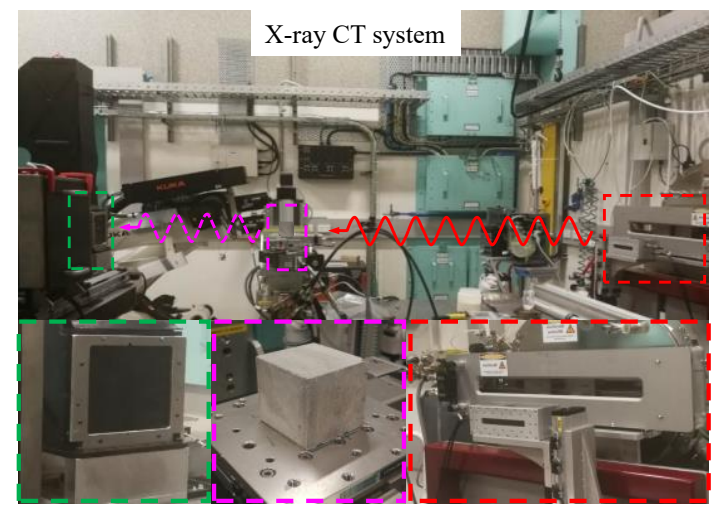

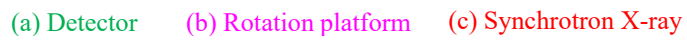

Fig. 3. Synchrotron X-ray computed tomography (CT) system. 


\section{Deformation and fracture characteristics}

Dynamic stress-strain curve and surface strain fields of concrete under impact velocity 16 $\mathrm{m} / \mathrm{s}$ and biaxial confinements $\left(\sigma_{1}, \sigma_{2}\right)$ of $(30,10) \mathrm{MPa}$ are shown in Figure 4. At $100 \mu$ s (i.e. pre-peak stage), axial compressive strain $\left(\mathrm{e}_{\mathrm{xx}}\right)$ localisation can be observed, which could be induced by the inhomogeneous deformation around aggregate. Lateral tensile strain $\left(\mathrm{e}_{\mathrm{yy}}\right)$ localisation can be also observed at $100 \mu \mathrm{s}$, indicating that matrix cracks are mainly induced by lateral tensile strain, verified by the result in literature [15]. At $170 \mu$ s (i.e. peak stage), compressive strain localisation areas exhibit more concentrated and lateral tensile strain localisation areas mainly develop along the impact direction. It is also observed that lateral tensile strain localisation areas are more likely to develop towards compressive strain localisation areas. The reason is that interfaces around aggregates with lower bond strength are more likely to form open crack, which is intensified due to the compressive strain concentration around aggregate and inconsistent deformation between aggregates and matrix. The interfaces will become weaker zones where main crack are prone to propagate.

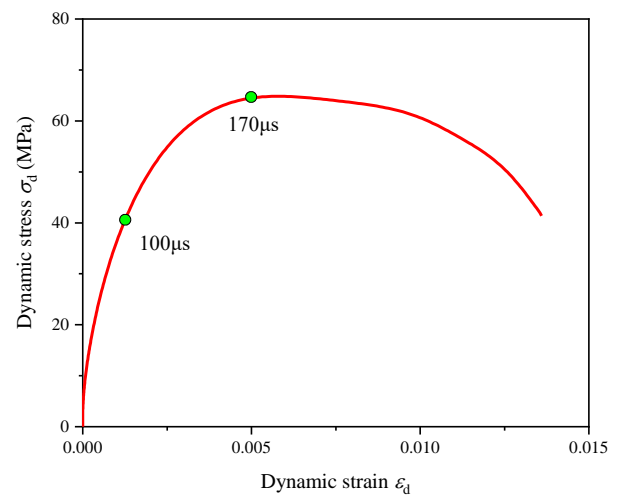

(a)
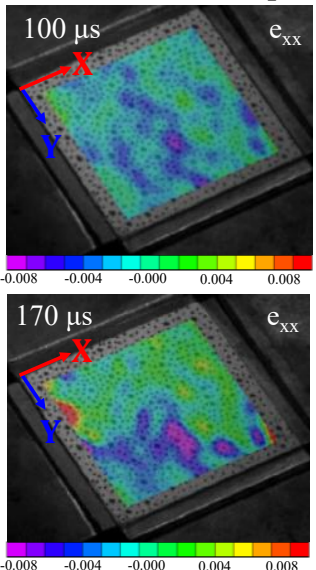

(b)
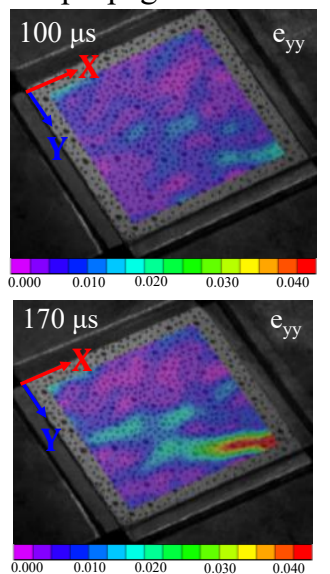

Fig. 4. (a) Dynamic stress-strain curve, and (b) synchronized strain fields obtained by 3D-DIC (exx: strain along $\mathrm{X}$ direction; eyy: strain along $\mathrm{Y}$ direction).

In order to verify the localised deformation and fracture behaviours in Figure 4, a simplified matrix-inclusion sample is fabricated with basalt cylinder as aggregate and same composition as matrix, shown in Figure 5.a. The loading condition is set as same as before. Results show that compressive strain is concentrated around aggregate and tensile crack will initiate at interface firstly at $100 \mu$ s (i.e. pre-peak stage). Main crack develops along the impact direction and coalescences with interfacial crack, which can well explain the reason why interface around aggregate under compressive strain localisation are more likely to induce the propagation of main cracks shown in Figure 4.

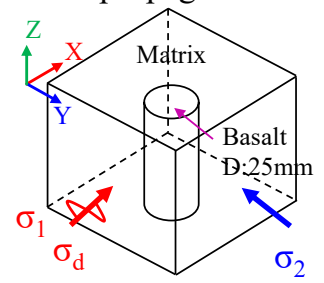

(a)

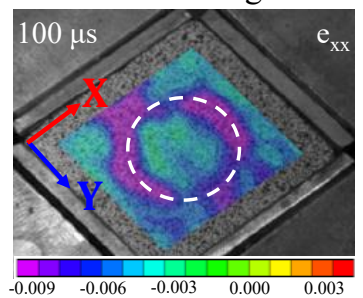

(b)

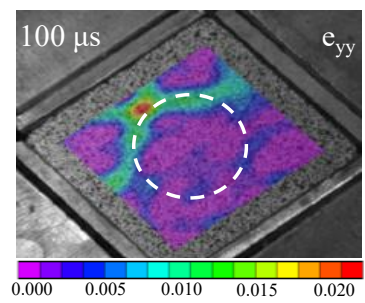

(c)

Fig. 5. Verification of strain fields around aggregate, (a) schematic of simplified matrix-inclusion sample, (b) axial strain fields and (c) lateral strain fields. 
Post-failure fractures obtained by synchrotron X-ray CT technique are shown in Figure 6. It can be seen that fractures, matrix and aggregates are shown in black, grey and white respectively due to the difference of X-ray attenuation with the variation of material density. From the 2D and 3D fracture CT images, it can be found that fractures mainly concentrate on top and bottom parts of specimen. In XY slice, the fractures propagate along the impact direction (i.e. X direction) and can be well corresponded with DIC results in Figure 4. In YZ slice, cracks are mainly parallel to $\mathrm{Y}$ direction, which indicates the cracks are mainly tensile cracks. However, in XZ slice, shear band can be also observed in addition to normal tensile cracks, indicating that maximum shear stress exists in the plane of max-min principal stress. In $Y Z$ and $X Z$ slices, transgranular cracks can be observed in the middle part of the specimen, especially in aggregates with major axis parallel to $Z$ direction. Compared with $Y Z$ and $X Z$ slices, interfacial cracks are dominated and less transgranular cracks can be observed in XY slice. Combined with the strain fields in Figure 4, it can be concluded that compressive strain will concentrate around aggregates in XY plane and induce tensile stress concentration along $\mathrm{Z}$ direction inside aggregates, which cause the initiation and propagation of transgranular cracks along $\mathrm{X}$ and $\mathrm{Y}$ directions.

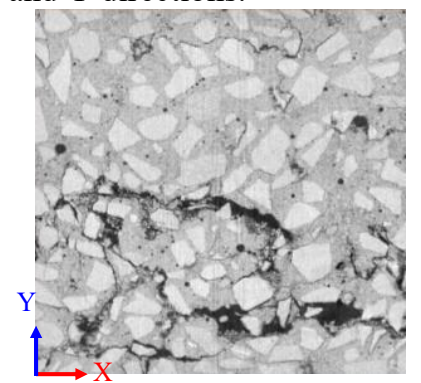

(a)

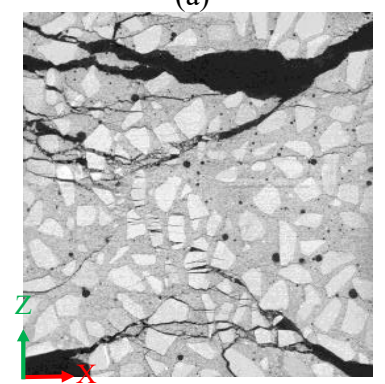

(c)

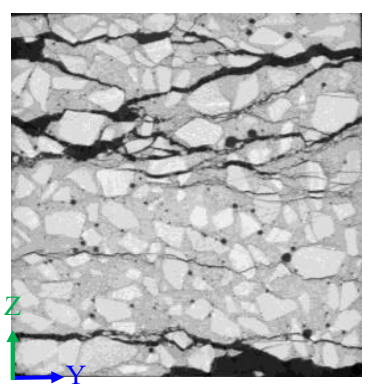

(b)

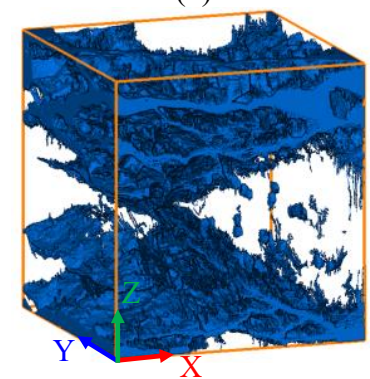

(d)

Fig. 6. CT images of concrete failure under dynamic loading and biaxial confinements: (a) XY slice, (b) YZ slice, (c) XZ slice and (d) 3D fracture volume rendering.

\section{Conclusions}

The dynamic deformation and fracture properties of ordinary concrete under biaxial confinements have been studied by Tri-HB system, 3D-DIC and X-ray CT techniques, and further verified by simplified matrix-inclusion sample. The results show that compressive strain localization will appear around aggregate during dynamic loadings due to the elastic mismatch and interfacial cracks will initiate firstly so that main cracks are more prone to propagate. Post failure CT images show that CT slices in different directions own distinct properties, which indicates the anisotropic fracturing behaviour of concrete due to the effect of biaxial confinement. Transgranular cracks can be observed in the central of specimen in 
$\mathrm{YZ}$ and $\mathrm{XZ}$ directions, which are related to the compressive strain concentration around aggregates in XY plane based on the strain field results

This work was supported financially by the Australian Research Council (LE150100058). The author would also appreciate the availability of Australian Synchrotron beamtime provided by Australia's Nuclear Science and Technology Organization (ANSTO) grant (NO: AS192/IMBL/14709). The first author is supported by the China Scholarship CouncilMonash University (CSC-Monash) project (NO: 201807090100).

\section{References}

1. H. K. H. Helmut Kupfer, R. Hubert, ACI Journal Proceedings, 66, 8 (1969)

2. J. Weerheijm, H.W. Reinhardt, Le. J. Phys. Col, 49, C3 (1988)

3. P. F. Liu, K. Liu, Q. B. Zhang, Constr Build Mater, 258 (2020)

4. E. Cadoni, C. Albertini, Advances in rock dynamics and applications (2011)

5. E. Cadoni, C. Albertini, M. Dotta, D. Forni, G. Riganti, C. Albertini, 94, EPJ Web of Conferences (2015).

6. W. R. Hu, K. Liu, D. O. Potyondy, Q. B. Zhang, Int. J. Rock Mech. Min. Sci, 134, (2020)

7. P. Forquin, G. Gary, F. Gatuingt, Int J Impact Eng, 35, 6 (2008)

8. Q. B. Zhang, J. Zhao, Int. J. Rock Mech. Min. Sci, 60, (2013)

9. H. Z. Xing, Q. B. Zhang, J. Zhao, Rock Mech. Rock Eng, 51, 3 (2018)

10. K. Liu, Q. B. Zhang, G. Wu, J. C. Li, J. Zhao, Rock Mech. Rock Eng, 52, 7 (2019)

11. Q. B. Zhang, K. Liu, G. Wu, J. Zhao, Handbook of Damage Mechanics (2021)

12. K. Liu, J. Zhao, G. Wu, A. Maksimenko, A. Haque, Q. B. Zhang, Int. J. Rock Mech. Min. Sci, 128, (2020)

13. G. L. Zhang, P. G. Ranjith, B. S. Wu, M. S. A. Perera, A. Haque, and D. Y. Li, Fuel, 255, (2019)

14. C. Chappard, A. Basillais, L. Benhamou, A. Bonassie, B. Brunet Imbault, N. Bonnet, F. Peyrin, Med Phys, 33, 9 (2006)

15. H. Z. Xing, Q. B. Zhang, D. Ruan, S. Dehkhoda, G. X. Lu, J. Zhao, Int J Impact Eng, 113, (2018) 